\title{
Demonstration of displacement-noise-free interferometry using bi-directional Mach-Zehnder interferometers
}

\author{
S Sato $^{1}$, K Kokeyama ${ }^{2}$, S Kawamura ${ }^{1}$, R L Ward ${ }^{3}$, A Nishizawa ${ }^{4}$, \\ $\mathrm{Y} \mathrm{Chen}^{5}$, A Pai ${ }^{5}$ and $\mathrm{K}$ Somiya ${ }^{5}$ \\ ${ }^{1}$ National Astronomical Observatory of Japan, Tokyo 181-8588, Japan \\ 2 Ochanomizu University, Tokyo 112-8610, Japan \\ 3 LIGO Project, California Institute of Technology, Pasadena, CA 91125, USA \\ ${ }^{4}$ Kyoto University, Kyoto 606-8501, Japan \\ ${ }^{5}$ Max-Planck-Institut für Gravitationsphysik, 14476 Potsdam, Germany \\ E-mail: sato.shuichi@nao.ac.jp
}

Received 5 November 2007, in final form 25 January 2008

Published 15 May 2008

Online at stacks.iop.org/CQG/25/114031

\begin{abstract}
We have demonstrated displacement- and frequency-noise-free laser interferometry (DFI) by partially implementing a recently proposed optical configuration using bi-directional Mach-Zehnder interferometers (MZIs). This partial implementation, the minimum necessary to be called DFI, has confirmed the essential feature of DFI: the combination of two MZI signals can be carried out in a way that cancels the displacement noise of the mirrors and beam splitters while maintaining gravitational-wave signals. The attained maximum displacement noise suppression was $45 \mathrm{~dB}$.
\end{abstract}

PACS numbers: $04.80 . \mathrm{Nn}$, 06.30.Ft, 95.55.Ym

(Some figures in this article are in colour only in the electronic version)

\section{Introduction}

The effects of gravitational wave (GW) and test-mass motion are essentially different. The practical application of this fact to the GW antenna was addressed only recently in [1,2]. As was shown theoretically, when each test mass in an $N$-test-mass array sends and receives light pulses from all other test masses, and if the array contains enough test masses $(N>(d+2)$, where $d$ is the number of spatial dimensions of the array), then there exist combinations of time delays which do not sense test-mass motions or timing noises, but do sense gravitational waves. We will call configurations that cancel both timing and displacement noises displacementnoise-free interferometry (DFI). 


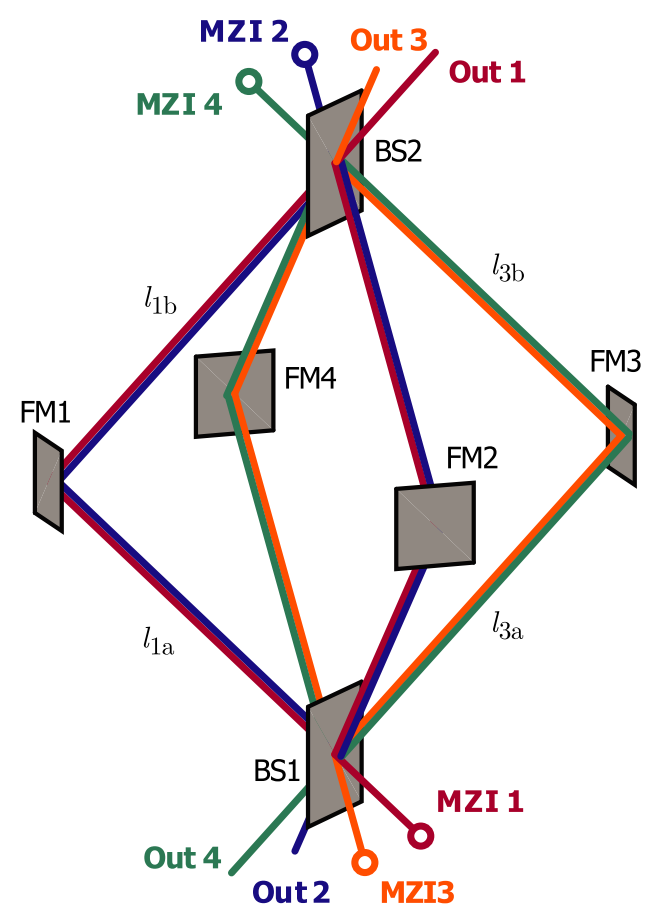

Figure 1. A schematic of three-dimensional DFI. The laser light propagates on the ridge line of the octahedron to form four simple MZIs. There are two beam splitters (BS) and four folding mirrors (FM) at the spatially symmetric apexes of the octahedron.

Recently, practical optical designs of DFI using laser interferometry in two and three dimensions have been proposed [3]. In these configurations, the conventional, equal-arm Mach-Zehnder interferometer (MZI) was used as a building block to eliminate laser noise. Four such MZIs were combined, in such a way that they form two pairs of counter-propagating MZIs. Within each pair, the two MZIs share the same beam splitters and folding mirrors; subtraction of their outputs balances out displacement noise from motions of the folding mirrors. The two pairs share the same beam splitters, which allows the elimination of beam splitter displacement noise. It should be mentioned that DFI configurations only work for nonzero frequencies, because at nearly zero frequency gravitational waves are indistinguishable from relative mirror motions.

In this paper, we study DFI experimentally using a single pair of counter-propagating MZIs and a single pair of independent MZIs. Two dedicated set-ups were used to demonstrate the elimination effects of both folding mirror and beam splitter displacement noises independently, retaining the response to gravitational waves.

\section{Partial demonstration of 3D DFI}

As shown in figure 1, the full configured 3D DFI is composed of two pairs of counterpropagating MZIs, for a total of four MZIs. MZIs of equal-arm length operated on the midfringe are insensitive to laser frequency noise, and by superimposing two counterpropagating MZIs on the same optical path, the displacements of the folding mirrors are sensed redundantly (once by each direction of the MZI) and can thus be unambiguously removed from the signal. An additional pair of counter-propagating MZIs can then be added, 
which shares beam splitters with the first pair, the second pair allows redundant sensing of beam splitter displacements. As discussed in [3], the signals from the four MZIs can be combined in such a way as to cancel the displacements of all the optics while retaining sensitivity to gravitational radiation.

In figure 1, the single pair of MZIs, composed of BS1, BS2, FM1, FM2, has equallength arms with folding mirrors located at the midpoint of each arm. As the folding mirrors experience some displacement noise, the light field incident on those mirrors is phase modulated. Because the folding mirrors are at the midpoint of the arms, and there is light traveling in both directions in the MZI, these displacement-noise-based phase modulations will arrive at outputs 1 and 2, simultaneously. Direct subtraction of outputs 1 and 2 then allows one to cancel the motion of the folding mirrors. Any signals which phase modulate the light and which do not arise at the midpoint will not arrive simultaneously at the outputs, and thus will not be fully cancelled. The response function of the displacement motion of the folding mirror $(\mathrm{FM} i)$ to the signal port is

$$
\begin{aligned}
& V_{1} \propto \mathrm{d} l_{\mathrm{FM} 1} \frac{\omega}{c} \mathrm{e}^{-\mathrm{i} l_{1 b} \Omega / c}-\mathrm{d} l_{\mathrm{FM} 2} \frac{\omega}{c} \mathrm{e}^{-\mathrm{i} l_{1 b} \Omega / c} \\
& V_{2} \propto \mathrm{d} l_{\mathrm{FM} 1} \frac{\omega}{c} \mathrm{e}^{-\mathrm{i} l_{1 a} \Omega / c}-\mathrm{d} l_{\mathrm{FM} 2} \frac{\omega}{c} \mathrm{e}^{-\mathrm{i} l_{1 a} \Omega / c},
\end{aligned}
$$

where $c$ is the speed of light, $\omega$ is the laser frequency and $\Omega$ is a Fourier frequency of the displacement motion. When the folding mirror is located at the exact center of the MZI arm (suppose $l_{i a}=l_{i b}=l$ ), all two signals display an identical frequency dependence in their response function which enables cancellation of signals due to displacement noise.

On the other hand, information about the beamsplitters is shared with another pair of MZIs, composed of BS1, BS2, FM3, FM4. Similar to the folding mirrors, phase modulations based on the beamsplitter (BS1) displacement will arrive at outputs 1 and 3, simultaneously. Therefore, direct subtraction of outputs 1 and 3 then allows one to cancel the motion of the beamsplitters. The response function of the displacement motion of the beamsplitter (BS1) to the signal port is

$$
\begin{aligned}
& V_{1} \propto \mathrm{d} l_{\mathrm{BS} 1} \frac{\omega}{c} \mathrm{e}^{-\mathrm{i}\left(l_{1 a}+l_{1 b}\right) \Omega / c}-\mathrm{d} l_{\mathrm{BS} 2} \frac{\omega}{c} \\
& V_{3} \propto \mathrm{d} l_{\mathrm{BS} 1} \frac{\omega}{c} \mathrm{e}^{-\mathrm{i}\left(l_{3 a}+l_{3 b}\right) \Omega / c}-\mathrm{d} l_{\mathrm{BS} 2} \cdot \frac{\omega}{c} .
\end{aligned}
$$

When the octahedron is spatially symmetric having the same beam propagation length (suppose $l_{i a}=l_{i b}=l$ ), all two signals display an identical frequency dependence in their response function which enables cancellation of signals due to displacement noise.

In contrast, as a substitute for exact simulation of the GW signals, one can generate a phase modulation signal at a location that is not the midpoint of an arm, and thus map the response of the interferometer to nonsymmetrically located signals, by observing the difference in arrival times between the two detection ports. In other words, even though DFI works because of the distributed nature of the phase modulation due to GWs, we can simulate it using pointmodulation (EOMs), which allows us to effectively measure Green's function. The response function to such a signal is as given in equation (1). So, as a result, the signal combination of MZI 1 and MZI 2 is given as

$$
H_{\text {sim GW }}= \pm \frac{\omega}{c}\left(\mathrm{e}^{-\mathrm{i} l_{1 a} \Omega / c}-\mathrm{e}^{-\mathrm{i} l_{1 b} \Omega / c}\right),
$$

which shows complete cancellation of the displacement motion of the folding mirror, while allowing simulated-GW signals to remain. 


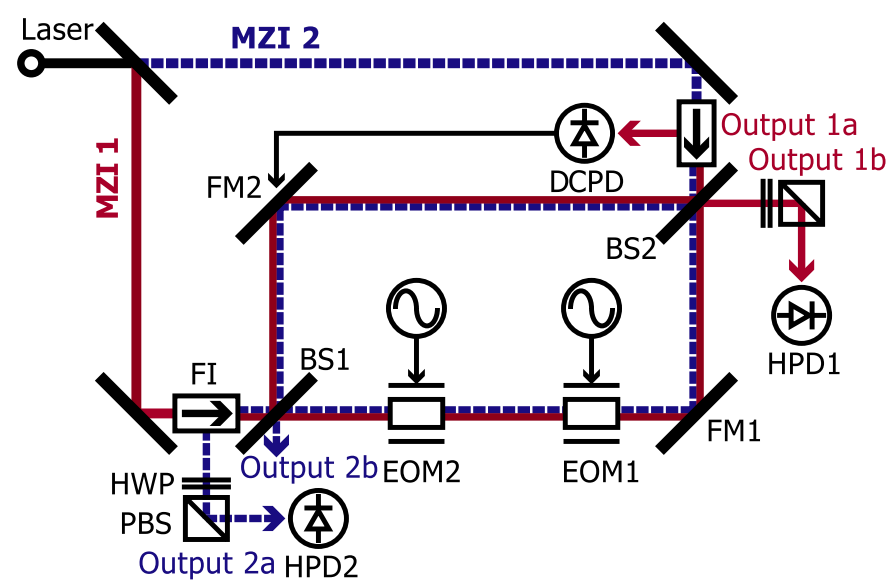

Figure 2. Practical setup for the partial demonstration of DFI. This is one pair of bi-directional MZIs, which is a part of 3D DFI, to confirm the cancellation of folding mirrors and the finite response to the GW signals.

\subsection{Cancellation of folding mirror}

For this experiment we have constructed a partial-DFI composed of a single pair of counterpropagating MZIs to demonstrate the cancellation properties of the DFI. The practical experimental setup is shown in figure 2. The laser source is a commercial solid-state Nd:YAG laser (Lightwave Model-126) yielding $100 \mathrm{~mW}$ at $1064 \mathrm{~nm}$. The output beam is split into two, and each beam introduced to two MZIs after passing through Faraday isolators (FI), which allow the detection of light exiting the counter-propagating MZI. The light paths of both MZIs are carefully adjusted and superposed on each other so that the two MZIs can exactly share the displacement noise and the effect of simulated-GW signals. The arm length of the MZIs affects the frequency where the MZI response to the GW signal is maximized; in this experiment, the arm lengths were chosen to be around $3.6 \mathrm{~m}$, to lower the peak frequency to several tens of $\mathrm{MHz}$. To enable high frequency operation, the phase modulation due to both displacement noise and a GW signal was simulated using electro-optic phase modulators located on one of the MZI arms. The displacement-noise simulator (EOM1) should be located at the exact center of the arm, so the position of EOM1 was carefully tuned, while the GW simulator (EOM2) was placed roughly $11 \mathrm{~cm}$ away from the BS1. The location of the EOM2 also determines the frequency of maximum sensitivity of DFI to the simulated-GW signal: a more asymmetrically placed EOM will give a lower peak frequency.

One of the output ports of MZI 1 (output 1a) was monitored with a de detector (DCPD) and an error signal created by subtracting a static offset; this signal was fed back to a PZTactuated folding mirror (FM2) after appropriate filtering to give a midfringe locking control. The control bandwidth was very low, around $400 \mathrm{~Hz}$, so that the simulated signal by EOMs would not be suppressed by the fringe control feedback loop in the higher, more interesting frequency band. Once the fringe of MZI 1 is controlled, that of MZI 2 is also automatically controlled because the two MZIs share common optical paths. Other output beams (output $1 \mathrm{~b}$ and $2 \mathrm{a}$ ) were received with high-speed photodetectors (New Focus 1611-AC) to monitor differential optical path length variations. The polarization optics (HWP: half wave plate and PBS: polarizing beam splitter) just in front of both high-speed detectors act as an optical attenuator, which serves as a gain compensator for imbalanced outputs of the two detectors. The signals from high-speed photodetectors HPD1 and HPD2 were summed with a power 


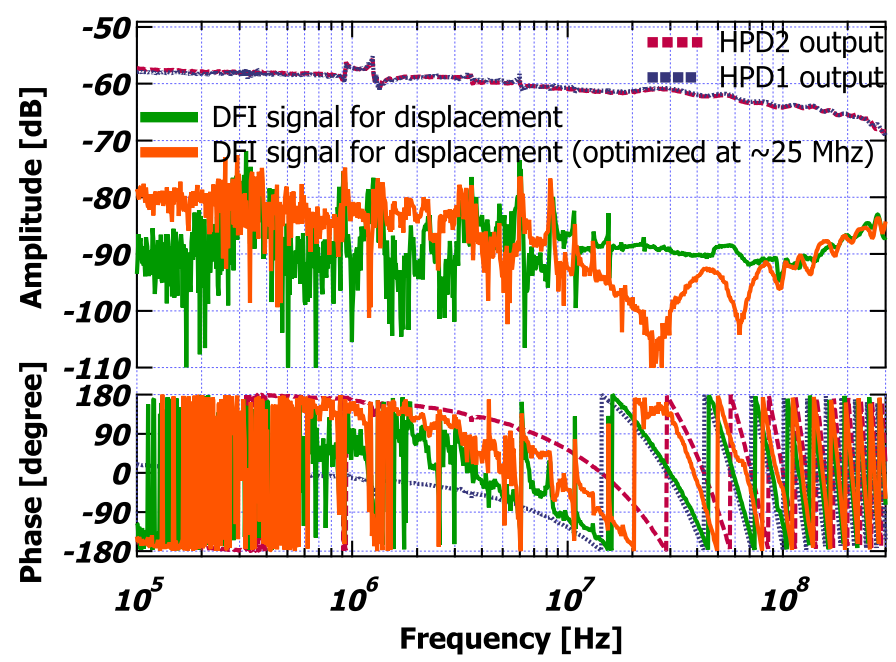

Figure 3. The transfer function from the displacement simulator to the interferometer output. The displacement information on HPD1 and HPD2 is significantly cancelled in the DFI signals.

combiner to produce DFI output signal, which was then monitored with a network analyzer (Anritsu MS4630B). The DFI features of bi-directional MZIs were demonstrated with a transfer function measurement from noise/signal simulators to DFI output. A swept-sine noise signal was provided by the internal oscillator of the network analyzer and was applied to the $\operatorname{EOM}(\mathrm{s})$.

The results for displacement-noise suppression are shown in figure 3. The transfer function of both detectors include the response functions of the simulating EOM, the photodetectors and optical and electronic phase delays from EOM through to the power combiner. Both amplitude and phase for the two signals were tuned to match each other using optical attenuators and path length adjustments so that the displacement-noise signal disappears in the DFI signal. About $30 \mathrm{~dB}$ of suppression of displacement noise was attained in a wide frequency region, while the maximum attained suppression was $45 \mathrm{~dB}$ at a particular frequency band, achieved when we tuned for maximum suppression in that region. Any imbalance between the two MZI signals determines the suppression ratio; it is believed that the subtly different frequency response of our two photodetectors, which cannot be compensated by simple optical attenuators or path length tuning, was the limiting factor here.

\subsection{Sensitivity to the simulated-GW signals}

On the other hand, for the simulated-GW signal, the measured response function is shown in figure 4. Single detector outputs show response function in amplitude similar to that for displacement noise; however, phase responses are different, which is what enables GW signal detection with DFI. The first peak frequency is around $40 \mathrm{MHz}$, corresponding to the scale of the instruments.

\subsection{Cancellation of beamsplitter}

For this experiment we have constructed another set of partial-DFI composed of a single pair of MZIs sharing a beamsplitter to demonstrate another cancellation property of the DFI. The 


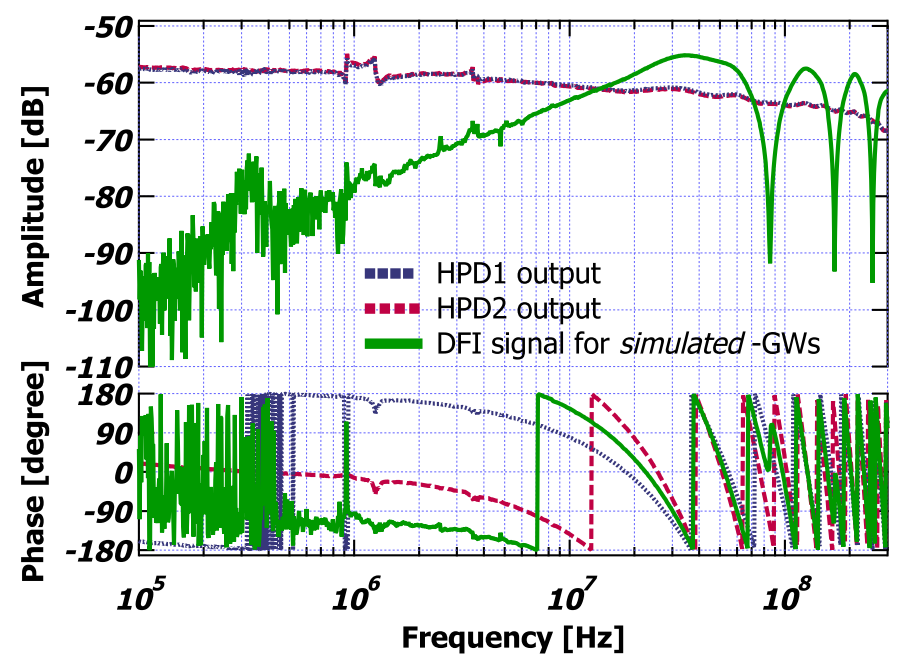

Figure 4. The response function to the simulated-GW signal. The identical signal subtraction of DFI to the displacement noise gives finite sensitivities to the GW-like phase modulations.

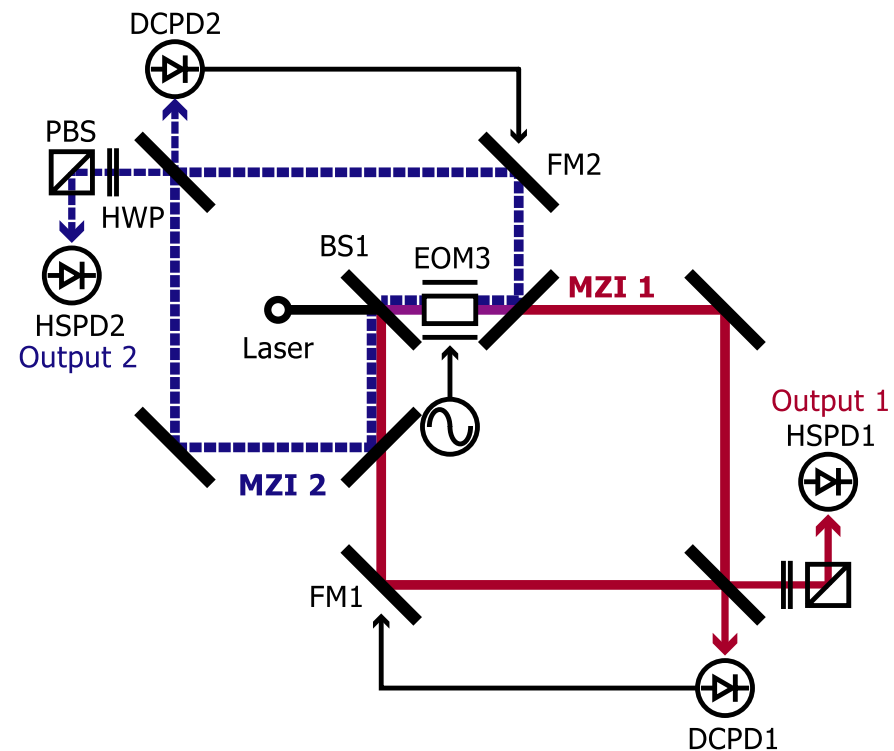

Figure 5. Another setup for the partial demonstration of DFI. This is one pair of two independent MZIs sharing beam splitter, which is a part of 3D DFI, to confirm the cancellation of beam splitter displacement.

practical experimental setup is shown in figure 5, which emulates MZI1 and MZI3 in figure 1 sharing an input beam splitter (BS1). The laser beam is introduced into beamsplitter BS1, then split into two to form independent MZIs. The arm length of the MZIs was chosen to be nearly the same as the previous setup of counter-propagating MZIs. The displacement-noise of the beam splitter was simulated by EOM3, which is located just after BS1. Displacement 


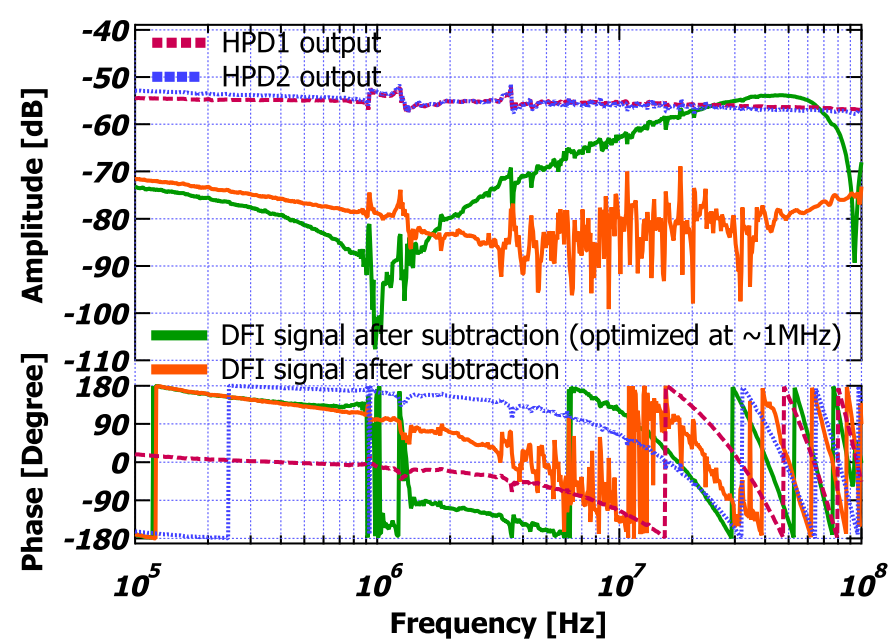

Figure 6. The transfer function from the displacement simulator to the interferometer output. The displacement information on the HPD1 and HPD2 is significantly cancelled in the DFI signals

noise simulation is identical to the previous experiment. One of the output ports of MZI 1 and MZI 2 was monitored with dc detectors (DCPD) and error signals were fed back to a PZT-actuated folding mirrors (FM1 and FM2) independently after appropriate filtering to give midfringe locking control. Other output beams were received with high-speed photodetectors to monitor differential optical path length variations. The polarization optics in front of both high-speed detectors again act as an optical attenuator, which serves as a gain compensator for imbalanced outputs of the two detectors. The signals from high-speed photodetectors HPD1 and HPD2 were summed with a power combiner to produce a DFI output signal, which was then monitored with a network analyzer.

The results for displacement-noise suppression are shown in figure 6. Both amplitude and phase for the two signals were tuned to match each other using optical attenuators and path length adjustments so that the displacement-noise signal disappears in the DFI signal. About $30 \mathrm{~dB}$ of suppression of displacement noise was attained in a wide frequency region, while the maximum attained suppression was $45 \mathrm{~dB}$ at a particular frequency band, achieved when we tuned for maximum suppression in that region.

\section{Summary}

We studied DFI experimentally using a single pair of counter-propagating MZIs and a single pair of independent MZIs, demonstrating the elimination of folding mirror and beam splitter displacement noise and the response to gravitational waves. The fundamental features of DFI were confirmed with these proof-of-principle experiments. The maximum attained suppression of displacement noise was $45 \mathrm{~dB}$ at a particular frequency band; it is believed that the subtly different frequency response of our two photodetectors was the limiting factor.

\section{Acknowledgments}

This research was partially supported by the Japanese Ministry of Education, Culture, Sports, Science and Technology under Grant-in-Aid for Scientific Research (B), No 18340070, 2006. 
RLW was supported by the US National Science Foundation under Cooperative Agreement No PHY-0107417. The research of YC, AP and KS is supported by the Alexander von Humboldt Foundation's Sofja Kovalevskaja Programme (funded by the German Ministry of Education and Research).

\section{References}

[1] Kawamura S and Chen Y 2004 Phys. Rev. Lett. 93211103

[2] Chen Y and Kawamura S 2006 Phys. Rev. Lett. 96231102

[3] Chen Y et al 2006 Phys. Rev. Lett. 97151103

[4] Sato S et al 2007 Phys. Rev. Lett. 98141101 